\title{
Gene expression profile for predicting survival of patients with meningioma
}

\author{
FENG CHEN $^{1 *}$, CHUN-XIANG XIANG $^{2 *}$, YI ZHOU $^{1}$, XIANG-SHENG AO $^{1}$, DA-QUAN ZHOU ${ }^{1}$, \\ PENG PENG $^{1}$, HAI-QUAN ZHANG ${ }^{1}$, HAN-DONG LIU ${ }^{1}$ and XING HUANG ${ }^{1}$ \\ ${ }^{1}$ Department of Neurosurgery, Xiangyang Central Hospital, Hubei University of Arts and Science, Xiangyang, Hubei 441021; \\ ${ }^{2}$ Department of Pathology, Wuhan Central Hospital, Wuhan, Hubei 430014, P.R. China
}

Received September 25, 2014; Accepted November 11, 2014

DOI: $10.3892 /$ ijo.2014.2779

\begin{abstract}
Current staging methods are inadequate for predicting the overall survival of meningioma. DNA microarray technologies improve the understanding of tumour progression. We analysed genome wide expression profiles of 119 meningioma samples from two previous published DNA microarray studies. The Cox proportional hazards regression models were applied to identify overall survival related gene signature. A total of 449 genes (109 upregulated and 340 downregulated) were identified as differentially expressed in meningioma. Among these differentially expressed genes, 37 genes were identified to be related to meningioma overall survival. Our 37-gene signature is closely associated with overall survival among patients with meningioma. This gene expression profile could provide an optimization of the clinical management and development of new therapeutic strategies for meningioma.
\end{abstract}

\section{Introduction}

Meningioma usually appears in the senior age of life and accounts to $22 \%$ of intracranial tumours in males and $38 \%$ in females (1). According to the WHO grading criteria, meningioma is divided into three grades: benign meningioma (grade I), atypical meningioma (grade II) and anaplastic meningioma (grade III). Anaplastic meningioma shows poorer prognosis than other common types of meningioma with a median overall survival time 2 years. Due to the difficulty of managing meningioma recurrence (2) and the low predictive powers of clinic pathological factors, biomarkers to identify

Correspondence to: Dr Feng Chen, Department of Neurosurgery, Xiangyang Central Hospital, Hubei University of Arts and Science, Xiangyang, Hubei 441021, P.R. China

E-mail: chenfenghuanle@163.com

*Contributed equally

Key words: gene expression, meningioma, prognosis, survival high-risk patients with a poor prognosis are strongly needed. From a previous study conducted in a total 124 samples from 105 patients using the high-resolution FISH-technology (iFISH), it is reported that the deletion of chromosome $1 \mathrm{p}$ may be an independent marker of meningioma recurrence and progression (3).

DNA microarray technology has enabled the simultaneous measurements of expression levels of thousands of genes in a group sample of tumour tissue, which could be used to provide prognostic information, or discriminate between various histologic subtypes. For the last decade, many research groups have used this technology to define prognostic gene signatures in many different tumour types, such as ovarian (4) breast (5), gastric (6), lung cancer (7) and neuroblastomas (8). The survival related gene signature might capture tumour progression status and could serve as a prerequisite to a more patient-tailored therapy.

Nevertheless, to the best of our knowledge, no study in which gene expression profiling was used to predict meningioma overall survival has been published yet. In the present study, we analysed genome-wide expression profiles of 119 meningioma samples using DNA microarray technologies. We applied Cox proportional hazards regression models and identified 37 prognostic genes related to meningioma overall survival, which could provide an optimization of the clinical management and development of new therapeutic strategies for meningioma.

\section{Materials and methods}

Data collection. Gene expression profiling datasets from two previously published studies analysed in the present study were referred as dataset 1 and dataset 2 . Dataset 1 consists of 51 samples, while dataset 2 consists of 68 samples.

For dataset 1 (9), there are 47 meningioma samples and 4 normal meninges. As previously discussed, the selection criteria of these samples include availability of enough tumour RNA, high quality of the extracted RNA and the representativeness of its cytogenetic profiles as defined by interphase fluorescence in situ hybridization (iFISH) within the whole series of tumours. Total RNA was extracted using RNeasy Mini kit (Quiagen, Valencia, CA, USA). The RNA integrity was assessed using the Agilent 2100 Bioanalyzer 
(Agilent Technologies, Inc., Palo Alto, CA, USA). The labelling process was performed according to the protocols from Affymetrix. Labelled RNA was hybridized to Human Genome U133A microarray, after quality checking on GeneChips Test 3 Arrays. Washing and scanning were performed using Fluidics Station 400 and GeneChip Scanner (Affymetrix).

For dataset 2 (10), tumour biopsies from 43 female and 25 male subjects with sporadic meningioma were selected from the UCLA Neuro-oncology Program Tissue Bank through institutional review board approved protocols. RNA was extracted from 20-50 mg tumour pieces using Qiagen ${ }^{\mathrm{TM}}$ (Qiagen) RNeasy Mini kits per manufacturer's protocols. The extracted total RNA was assessed for integrity using the 2100 Bioanalyzer by Agilent Technologies. Total RNA $(1 \mu \mathrm{g})$ was used for single-round biotinylated probe synthesis using the Affymetrix Array Station device made by Caliper Life Sciences (Hopkinton, MA, USA) by the manufacturer's protocols. Labelled and sheared cRNA was manually applied to Affymetrix Human Genome U133 Plus 2.0 Arrays (Affymetrix). All microarrays were scanned using the Affymetrix GeneChip scanner 3000.

Data processing. We retrieved the raw fluorescence intensity data within CEL files from NCBI GEO database with accession number GSE43290 (dataset 1) and GSE16581 (dataset 2). The raw datasets were preprocessed with gcrma algorithm, as implemented with $\mathrm{R}$ packages from Bioconductor (http:// www.bioconductor.org). The gcrma algorithm (11) adjusts for the background intensities in Affymtrix array data by including optical noise and non-specific binding (NSB). It then converts background adjusted probe intensities to expression measures using the same normalization and summarization methods implanted by the robust multiarray average (RMA) (12) algorithm. Because two different microarray platforms were used in these datasets, the probe sets had to be matched to identical genes. Based on the latest official symbol annotation provided by the manufacturer, we developed a Perl script to match probe sets among datasets 1 and 2 . This process produced a total of 13,879 genes on both the two Affymetrix microarray systems: Human Genome U133A microarray and Human Genome U133 Plus 2.0 Arrays.

Microarray and statistical analysis. We then used the statistical software programs R, the R-package, limma (13), for the analysis of gene expression microarray data from dataset 1 . This algorithm uses linear models, as well as Empirical Bayesian methods, for analysing designed experiments and assessment of differential expression. The Cox proportional hazards regression analysis was conducted to identify survival related genes. Survival analysis was performed using the R-package 'survival' (14). Overall survival (OS) was analysed using the Kaplan-Meier product-limit method and the significance of the variables was measured by the log-rank test. Hierarchical clustering based on Euclidean distance and Ward's clustering method were used to show the expression patterns of survival-related genes in dataset 2 . We further analysed the Gene Ontology and canonical pathways with the use of DAVID (The Database for Annotation, Visualization and Integrated Discovery) tools (15).
Table I. Clinical characteristics of the patients with meningioma tumours.

\begin{tabular}{lcc}
\hline Demographics & Dataset $1, \mathrm{n}=51$ & Dataset 2, $\mathrm{n}=68$ \\
\hline $\begin{array}{l}\text { Survival time, days } \\
\text { Median }\end{array}$ & NA & 1,726 \\
Range & NA & $19-3,387$ \\
Age, years & & \\
Median & 65 & 64 \\
Range & $23-84$ & $32-89$ \\
Gender & & \\
Male & 16 & 25 \\
Female & 35 & 43 \\
Grade & & \\
I & 33 & 43 \\
II & 12 & 19 \\
III & 2 & 6 \\
Normal & 4 & NA \\
\hline
\end{tabular}

\section{Results}

Clinical characteristics. The clinical characteristics of samples in dataset 1 and 2 are summarized in Table I. We used dataset 1 for the differential expression analysis to pre-select the survival gene candidates. Within this dataset, a total of 51 patients (16 males and 35 females with a median age 65 years; range, 23-84 years) were analysed. All tumours were diagnosed and classified according to WHO criteria: 33 patients $(64.71 \%)$ were diagnosed as grade I, 12 patients $(23.53 \%)$ as grade II, and 2 patients (3.92\%) as grade III. On the other hand, we used dataset 2 for the identification of survival related gene expression profile. Among the 68 samples ( 25 males and 43 females with a median age 64 years; range, $32-89$ years), 43 patients (63.24\%) were diagnosed as grade I, 19 patients (27.94\%) as grade II and 6 patients ( $8.82 \%)$ as grade III. The median survival time is 1,726 days with range of 19-3,387 days.

Identification of a 37-gene signature for meningioma prognosis. Our hypothesis is that the genes involved in the progression of meningioma may also contribute to the prediction of prognosis. Therefore, we firstly conducted differential expression analysis in dataset 1 using Affymetrix Human Genome U133A microarray. A total of 449 genes (109 upregulated and 340 downregulated) were identified as significantly differentially expressed in meningioma. As shown in Fig. 1, the threshold is set as a log-odds value of $>4.6$ (99\% probability that the gene is differentially expressed) and a fold-change $>2$-fold. The 449 genes were used as a discovery set for the identification of survival related profiles in meningioma.

To investigate the prognostic potential of these genes, we prepared dataset 2 . To deal with cross-platform microarray data appropriately, we mapped the probe set IDs from two microarray platforms to official symbols. A univariate Cox proportional hazard model showed that expression levels of 51 probes (representing 37 non-redundant genes) were 
Table II. Thirty-seven genes composing the survival related profile.

\begin{tabular}{|c|c|c|}
\hline Symbol & Chromosome location & Description \\
\hline ARHGDIA & $17 q 25.3$ & Rho GDP dissociation inhibitor (GDI) $\alpha$ \\
\hline ATP6V1G2 & $6 \mathrm{p} 21.3$ & ATPase, $\mathrm{H}^{+}$transporting, lysosomal $13 \mathrm{kDa}, \mathrm{V} 1$ subunit G2 \\
\hline BCAS1 & $20 \mathrm{q} 13.2$ & Breast carcinoma amplified sequence 1 \\
\hline CD200 & $3 q 12-q 13$ & CD200 molecule \\
\hline CNOT3 & $19 q 13.4$ & CCR4-NOT transcription complex, subunit 3 \\
\hline CSF3 & $17 q 11.2-q 12$ & Colony stimulating factor 3 (granulocyte) \\
\hline CSGALNACT2 & $10 \mathrm{q} 11.21$ & Chondroitin sulfate $\mathrm{N}$-acetylgalactosaminyltransferase 2 \\
\hline CTBP1 & $4 \mathrm{p} 16$ & C-terminal binding protein 1 \\
\hline CXCL3 & $4 q 21$ & Chemokine (C-X-C motif) ligand 3 \\
\hline EIF2S3 & Xp22.2-p22.1 & Eukaryotic translation initiation factor 2 , subunit $3 \gamma, 52 \mathrm{kDa}$ \\
\hline EIF5A & 17p13-p12 & Eukaryotic translation initiation factor $5 \mathrm{~A}$ \\
\hline ELAVL1 & $19 \mathrm{p} 13.2$ & ELAV (embryonic lethal, abnormal vision, Drosophila)-like 1 (Hu antigen R) \\
\hline ERG & $21 \mathrm{q} 22.3$ & v-ets erythroblastosis virus E26 oncogene homolog (avian) \\
\hline ESR1 & $6 q 25.1$ & Estrogen receptor 1 \\
\hline FKBP8 & $19 \mathrm{p} 12$ & FK506 binding protein $8,38 \mathrm{kDa}$ \\
\hline FUS & $16 \mathrm{p} 11.2$ & Fused in sarcoma \\
\hline HBD & $11 \mathrm{p} 15.5$ & Hemoglobin, $\delta$ \\
\hline HLA-F & $6 \mathrm{p} 21.3$ & Major histocompatibility complex, class I, F \\
\hline HMGN1 & $21 \mathrm{q} 22.2$ & High mobility group nucleosome binding domain 1 \\
\hline HNRNPR & $1 \mathrm{p} 36.12$ & Heterogeneous nuclear ribonucleoprotein $\mathrm{R}$ \\
\hline IL8 & $4 q 13-q 21$ & Interleukin 8 \\
\hline L1CAM & $\mathrm{Xq} 28$ & L1 cell adhesion molecule \\
\hline LOC 100129518 & $6 q 25.3$ & Uncharacterized LOC100129518 \\
\hline MAFG & $17 \mathrm{q} 25.3$ & v-maf musculoaponeurotic fibrosarcoma oncogene homolog G (avian) \\
\hline MBP & $18 \mathrm{q} 23$ & Myelin basic protein \\
\hline NUPL1 & $13 q 12.13$ & Nucleoporin like 1 \\
\hline PAPOLA & $14 q 32.31$ & Poly(A) polymerase $\alpha$ \\
\hline PRRC2B & $9 q 34.13$ & Proline-rich coiled-coil 2B \\
\hline SCAF4 & $21 \mathrm{q} 22.1$ & SR-related CTD-associated factor 4 \\
\hline SENP3 & $17 \mathrm{p} 13$ & SUMO1/sentrin/SMT3 specific peptidase 3 \\
\hline SET & $9 q 34$ & SET nuclear oncogene \\
\hline SETP4 & $\mathrm{Xq} 21.1$ & SET pseudogene 4 \\
\hline SOD2 & $6 q 25.3$ & Superoxide dismutase 2 , mitochondrial \\
\hline SOX10 & $22 q 13.1$ & SRY (sex determining region Y)-box 10 \\
\hline SUMO2 & $17 q 25.1$ & SMT3 suppressor of mif two 3 homolog 2 (S. cerevisiae) \\
\hline USP46 & $4 q 12$ & Ubiquitin specific peptidase 46 \\
\hline WBP4 & $13 q 14.11$ & WW domain binding protein 4 \\
\hline
\end{tabular}

correlated with overall survival time $(\mathrm{P}<0.05)$ (Table II). As shown in Fig. 2, the 37-gene signature was the dominant characteristics that permitted the stratification of individuals into cancerous and normal groups. To determine whether the gene expression profiles could accurately predict overall survival, hierarchical clustering was used to classify all of the samples from dataset 2 into two groups as high- and low-risk groups (Fig. 3A). Kaplan-Meier analysis demonstrated that the highand low-risk groups were significantly different in their overall survival $(\mathrm{P}<0.01)$ (Fig. 3B).
Characterization of survival related profile. We conducted gene set enrichment analysis to understand the biological characteristics of the 37-gene signature. The gene signature includes three oncogenes, FUS (FUS RNA binding protein), ERG (v-ets avian erythroblastosis virus E26 oncogene homolog) and SET (SET nuclear proto-oncogene); two cell differentiation markers, CD200 (CD200 molecule) and L1CAM (L1 cell adhesion molecule); five transcription factors, ESR1 (estrogen receptor 1), MAFG (v-maf avian musculoaponeurotic fibrosarcoma oncogene homolog G), CNOT3 


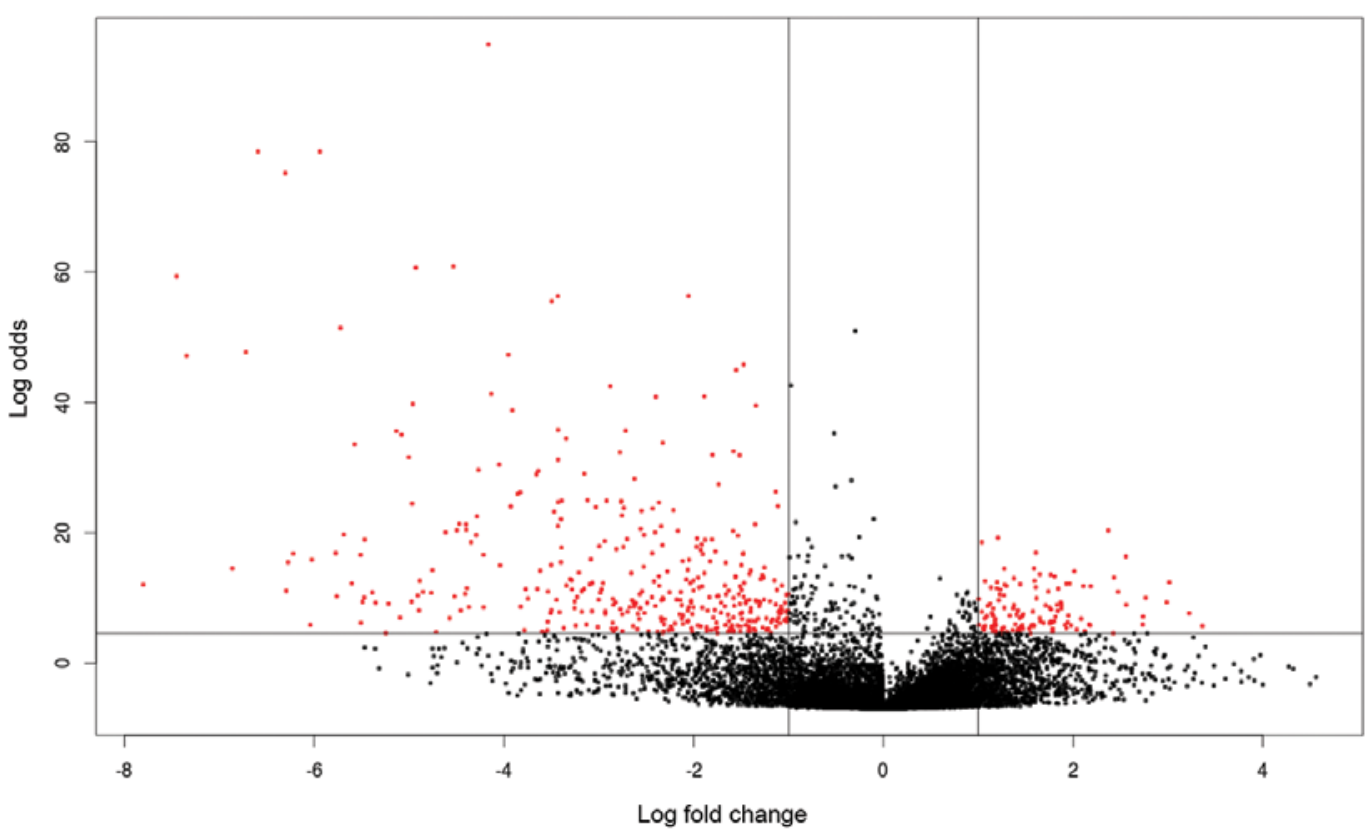

Figure 1. Plot of log-fold changes vs. log-odds of differential expression. The $\mathrm{x}$-axis indicates the log 2 value of fold-change between the two conditions. The log-odds (or B value) on the y-axis is the odds (or probability) that the gene is differentially expressed. The red dots located in the upper left and right square are genes that have a log-odds score of 4.6 or more, have a fold-change $>2$-fold, and are identified as significantly differentially expressed in meningioma tumours.

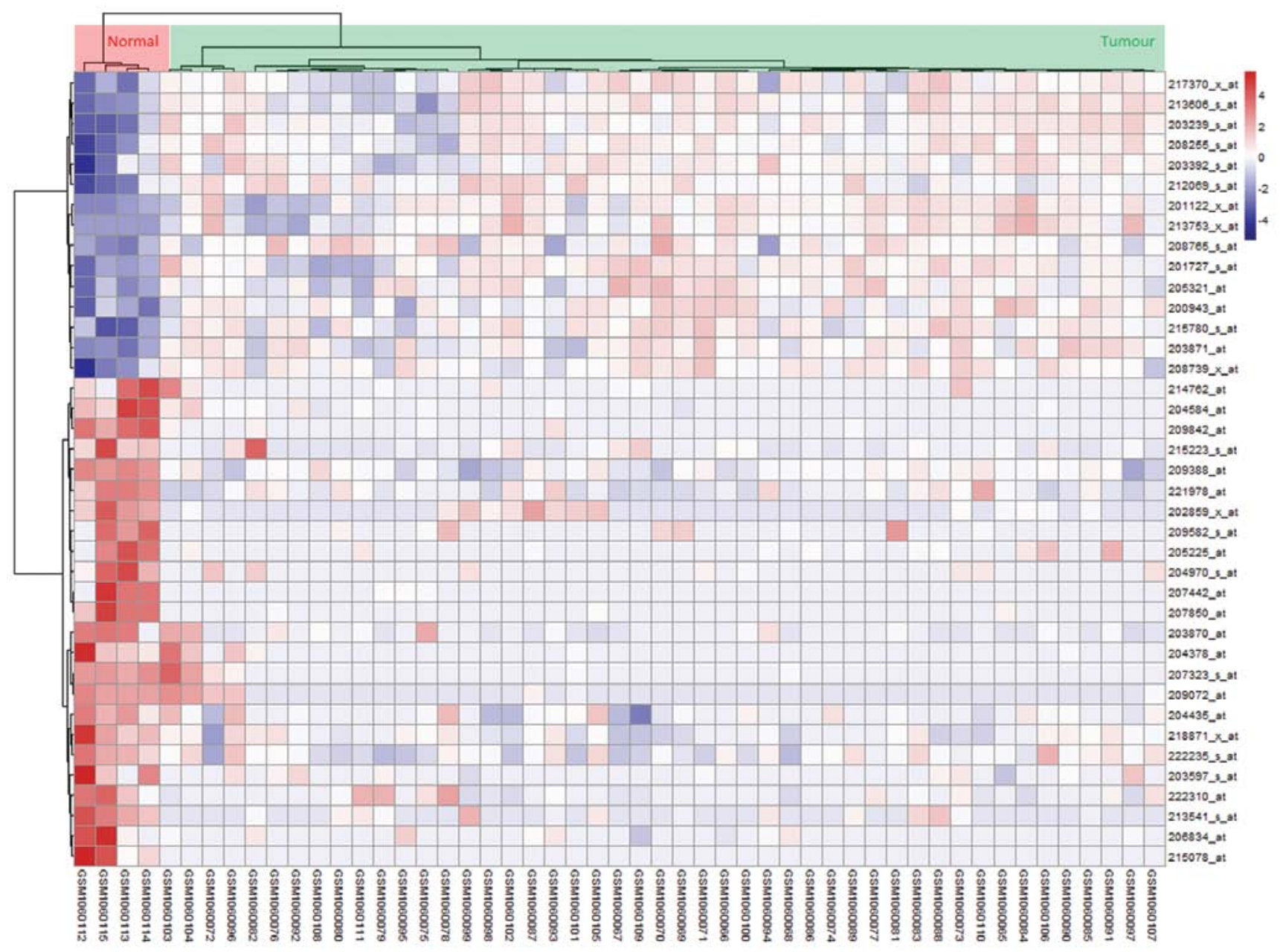

Figure 2. Hierarchical clustering analysis of dataset 1 using the 37-gene signature. The data are presented in matrix format, in which rows represent individual genes and columns represent each sample. The genes are labelled with Affymetrix probe set IDs, while the samples are labelled with NCBI GEO accession numbers. 

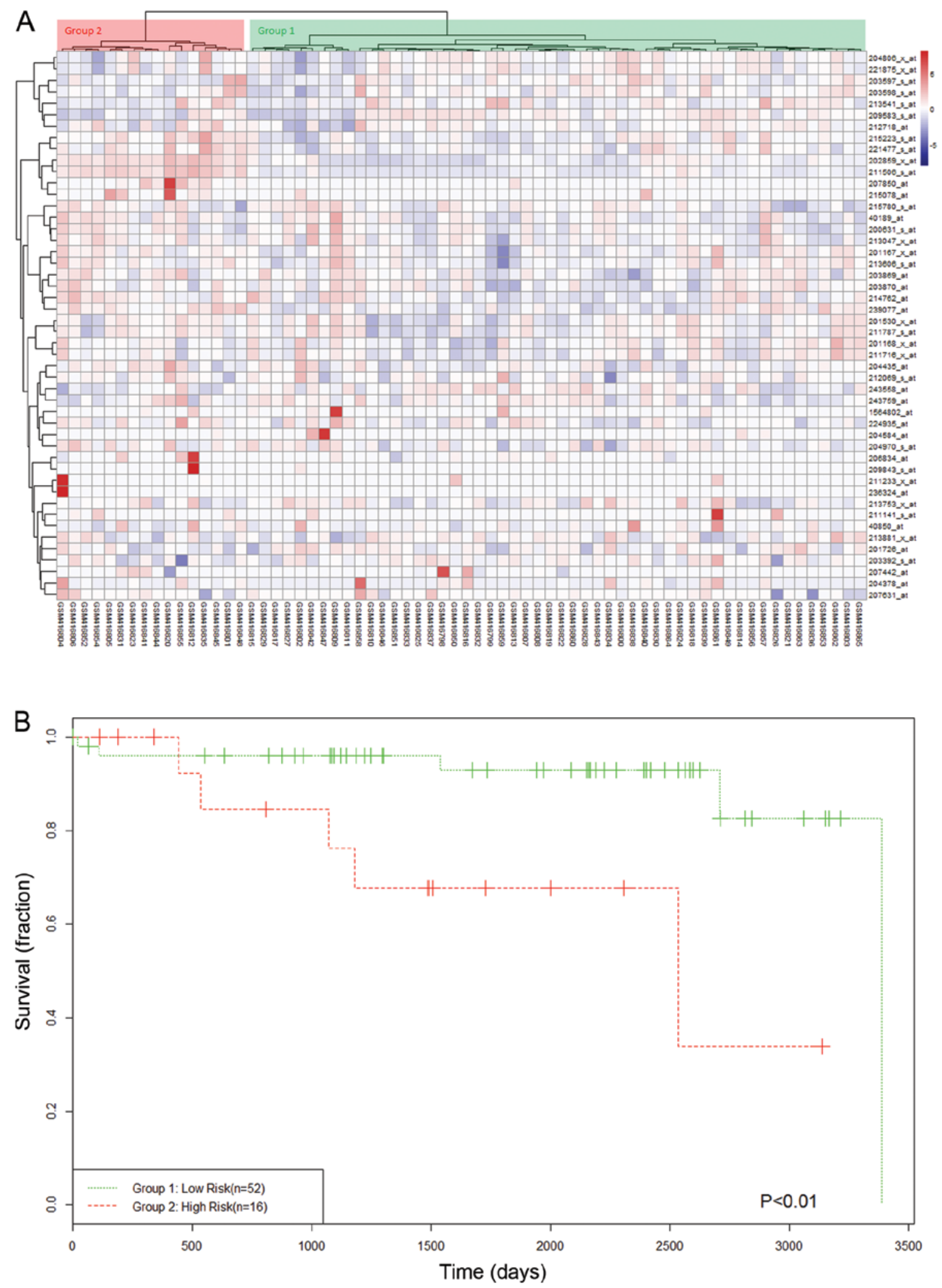

Figure 3. Application of the 37-gene signature for meningioma tumours. (A) Clustering of the patient samples into high-risk (red) and low-risk (green) groups using the 37-gene signature. The data are presented in matrix format, in which rows represent individual genes and columns represent each sample. The genes are labelled with Affymetrix probe set IDs, while the samples are labelled with NCBI GEO accession numbers; (B) Kaplan-Meier overall survival analysis of the high- and low-risk groups according to the hierarchical clustering result.

(CCR4-NOT transcription complex, subunit 3), SOX10 (sex determining region Y-box 10) and ERG.

To characterize the gene list based on GO classification on 'biological process', 'molecular function' and 'cellular component', we examined in which categories the gene signature was significantly enriched. The 37 genes were significantly (P-value $<0.05$ ) enriched in 16 GO categories. In the biological process class, the genes were notably enriched 


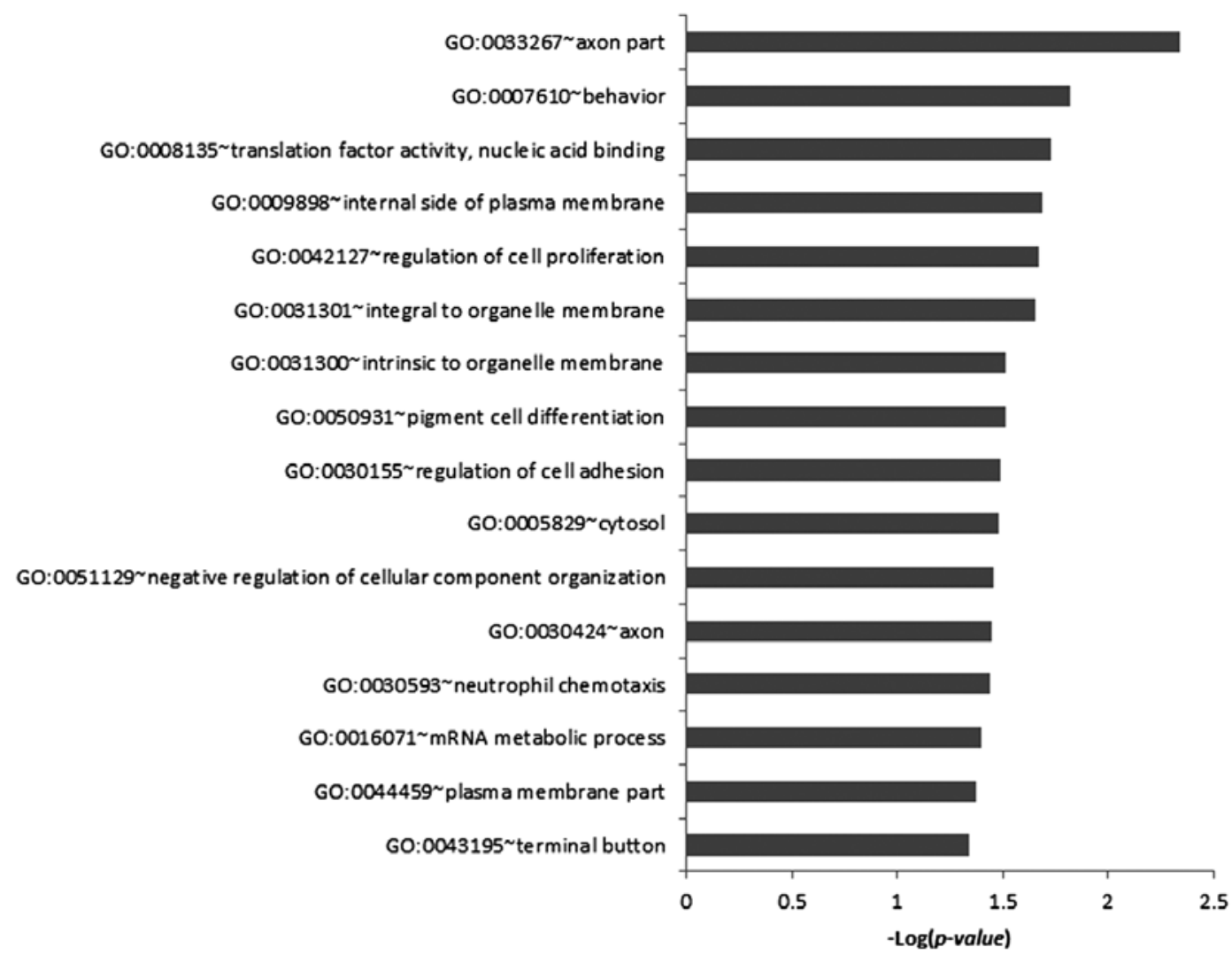

Figure 4. Biological characteristics of the 37 overall survival-related genes. The significantly enriched Gene Ontology (GO) categories in gene set enrichment analysis.

in behaviour (GO:0007610), regulation of cell proliferation (GO:0042127), pigment cell differentiation (GO:0050931), regulation of cell adhesion (GO:0030155), negative regulation of cellular component organization (GO:0051129), neutrophil chemotaxis (GO:0030593) and mRNA metabolic process (GO:0016071). In cellular component class, the genes were significantly enriched in axon part (GO:0033267), internal side of plasma membrane (GO:0009898), integral to organelle membrane (GO:0031301), intrinsic to organelle membrane (GO:0031300), cytosol (GO:0005829), axon (GO:0030424), plasma membrane part (GO:0044459) and terminal button (GO:0043195). In the molecular function class, the genes were only enriched in translation factor activity, nucleic acid binding (GO:0008135) (Fig. 4).

However, we could not identify any pathway that the 37 genes were significantly enriched in.

\section{Discussion}

In the present study, we identified a 37-gene prognostic signature for meningioma patients across two types of microarray expression profiling datasets by using Cox proportional hazard models. This gene signature was independently predictive of survival and outperforming current pathological staging criteria.

Our survival gene signature consists of genes that showed prognostic potential in other cancer types. For instance, the gene ARHGDIA [Rho GDP dissociation inhibitor (GDI) alpha] is associated with mesothelioma $(16,17)$ prognosis and could serve as an independent prognostic factor in hepatocellular carcinoma (18). Another example is the gene CD200. CD200 is expressed in most PCM (plasma cell myeloma) cases and its expression level remains stable even during the treatment process, which could serve as a useful marker for the prognosis of PCM $(19,20)$. It is also reported that CD200 could be a new prognostic factor in acute myeloid leukaemia (21). ESR1, a ligand-activated transcription factor, involves in pathological processes of breast (22-25), prostate (26) and non-small cell lung cancer (27).

Surprisingly, from the differential expression analysis, a small number of genes that upregulated or downregulated were identified in meningioma comparing to normal meninges. This suggests that meningioma and normal meninges may remain largely homogeneous at global gene expression level. However, the identified differentially expressed genes appear to be clinically and even biologically important, which could ultimately influence the prognostic outcome.

To the best of our knowledge, the present study is the first to identify a prognostic signature for meningioma prognosis based on genome wide expression profiling technologies. However, because most of the current public accessible meningioma data lack sufficient follow-up information, we could not evaluate the prognostic predictive power in other independent datasets. For future work, we will continuously work on optimizing the prognosis model by integrating more meningioma datasets.

In conclusion, these results suggest that the 37-gene signature could serve as a useful tool to predict the meningioma 
progression and survival. To apply in the clinical practice, a prospective multi-centre study is still needed to improve the predictive ability and reliability of the gene expression profile. Nevertheless, the survival related gene expression profile could provide an optimization of the clinical management and development of new therapeutic strategies for meningioma.

\section{References}

1. Claus EB, Bondy ML, Schildkraut JM, Wiemels JL, Wrensch M and Black PM: Epidemiology of intracranial meningioma. Neurosurgery 57: 1088-1095, 2005.

2. Pasquier D, Bijmolt S, Veninga T, et al: Atypical and malignant meningioma: outcome and prognostic factors in 119 irradiated patients. A multicenter, retrospective study of the Rare Cancer Network. Int J Radiat Oncol Biol Phys 71: 1388-1393, 2008.

3. Linsler S, Kraemer D, Driess C, et al: Molecular biological determinations of meningioma progression and recurrence. PloS One 9: e94987, 2014

4. Konstantinopoulos PA, Spentzos D and Cannistra SA: Geneexpression profiling in epithelial ovarian cancer. Nat Clin Pract Oncol 5: 577-587, 2008.

5. van 't Veer LJ, Dai H, van de Vijver MJ, et al: Gene expression profiling predicts clinical outcome of breast cancer. Nature 415 : 530-536, 2002

6. Motoori M, Takemasa I, Yano M, et al: Prediction of recurrence in advanced gastric cancer patients after curative resection by gene expression profiling. Int J Cancer 114: 963-968, 2005.

7. Chen $\mathrm{HY}, \mathrm{Yu} \mathrm{SL}$, Chen $\mathrm{CH}$, et al: A five-gene signature and clinical outcome in non-small-cell lung cancer. N Engl J Med 356: 11-20, 2007.

8. Schramm A, Schulte JH, Klein-Hitpass L, et al: Prediction of clinical outcome and biological characterization of neuroblastoma by expression profiling. Oncogene 24: 7902-7912, 2005.

9. Tabernero MD, Maillo A, Gil-Bellosta CJ, et al: Gene expression profiles of meningiomas are associated with tumor cytogenetics and patient outcome. Brain Pathol 19: 409-420, 2009.

10. Lee Y, Liu J, Patel S, et al: Genomic landscape of meningiomas. Brain Pathol 20: 751-762, 2010.

11. Wu Z, Irizarry RA, Gentleman R, Martinez-Murillo F and Spencer F: A model-based background adjustment for oligonucleotide expression arrays. J Am Stat Assoc 99: 909-917, 2004

12. Irizarry RA, Hobbs B, Collin F, et al: Exploration, normalization, and summaries of high density oligonucleotide array probe level data. Biostatistics 4: 249-264, 2003.
13. Smyth GK: Limma: linear models for microarray data. In: Bioinformatics and Computational Biology Solutions Using $R$ and Bioconductor. Springer, New York, pp397-420, 2005.

14. Therneau TM and Grambsch PM: Modeling Survival Data: Extending the Cox Model. Springer, New York, 2000.

15. Huang da W, Sherman BT and Lempicki RA: Systematic and integrative analysis of large gene lists using DAVID bioinformatics resources. Nat Protoc 4: 44-57, 2009.

16. Gordon GJ, Bueno R and Sugarbaker DJ: Genes associated with prognosis after surgery for malignant pleural mesothelioma promote tumor cell survival in vitro. BMC Cancer 11: 169, 2011.

17. Gordon GJ, Dong L, Yeap BY, et al: Four-gene expression ratio test for survival in patients undergoing surgery for mesothelioma. J Natl Cancer Inst 101: 678-686, 2009.

18. Li W, Wang H, Jin X and Zhao L: Loss of RhoGDI is a novel independent prognostic factor in hepatocellular carcinoma. Int J Clin Exp Pathol 6: 2535-2541, 2013.

19. Douds JJ, Long DJ, Kim AS and Li S: Diagnostic and prognostic significance of CD200 expression and its stability in plasma cell myeloma. J Clin Pathol 67: 792-796, 2014.

20. Alapat D, Coviello-Malle J, Owens R, et al: Diagnostic usefulness and prognostic impact of CD200 expression in lymphoid malignancies and plasma cell myeloma. Am J Clin Pathol 137: 93-100, 2012.

21. Tonks A, Hills R, White P, et al: CD200 as a prognostic factor in acute myeloid leukaemia. Leukemia 21: 566-568, 2007.

22. Caizzi L, Ferrero G, Cutrupi S, et al: Genome-wide activity of unliganded estrogen receptor-alpha in breast cancer cells. Proc Natl Acad Sci USA 111: 4892-4897, 2014.

23. Gao QG, Chan HY, Man CW and Wong MS: Differential ERalpha-mediated rapid estrogenic actions of ginsenoside Rg1 and estren in human breast cancer MCF-7 cells. J Steroid Biochem Mol Biol 141: 104-112, 2014.

24. Martinez-Galan J, Torres-Torres B, Nunez MI, et al: ESR1 gene promoter region methylation in free circulating DNA and its correlation with estrogen receptor protein expression in tumor tissue in breast cancer patients. BMC Cancer 14: 59, 2014

25. Toy W, Shen Y, Won H, et al: ESR1 ligand-binding domain mutations in hormone-resistant breast cancer. Nat Genet 45 : 1439-1445, 2013.

26. Gu Z, Wang G and Chen W: Estrogen receptor alpha gene polymorphisms and risk of prostate cancer: a meta-analysis involving 18 studies. Tumour Biol 35: 5921-5930, 2014.

27. Atmaca A, Al-Batran SE, Wirtz RM, et al: The validation of estrogen receptor $1 \mathrm{mRNA}$ expression as a predictor of outcome in patients with metastatic non-small cell lung cancer. Int J Cancer 134: 2314-2321, 2014 